\title{
JMJD1A Represses the Development of Cardiomyocyte Hypertrophy by Regulating the Expression of Catalase
}

\author{
Rongjia Zang, ${ }^{1}$ Qingyun Tan, ${ }^{1}$ Fanrong Zeng, ${ }^{1}$ Dongwei Wang, ${ }^{1}$ Shuang $Y u^{2}$ \\ and Qingdong Wang $\mathbb{D}^{1}$ \\ ${ }^{1}$ Department of Anesthesiology, First Affiliated Hospital of Jiamusi University, Heilongjiang Province, China \\ ${ }^{2}$ Department of Cardiology, First Affiliated Hospital of Jiamusi University, Heilongjiang Province, China \\ Correspondence should be addressed to Qingdong Wang; 3190013@qq.com
}

Received 19 January 2020; Revised 15 April 2020; Accepted 27 April 2020; Published 13 May 2020

Academic Editor: Stavros Baloyannis

Copyright (C) 2020 Rongjia Zang et al. This is an open access article distributed under the Creative Commons Attribution License, which permits unrestricted use, distribution, and reproduction in any medium, provided the original work is properly cited.

\begin{abstract}
The histone demethylase JMJD family is involved in various physiological and pathological functions. However, the roles of JMJD1A in the cardiovascular system remain unknown. Here, we studied the function of JMJD1A in cardiac hypertrophy. The mRNA and protein levels of JMJD1A were significantly downregulated in the hearts of human patients with hypertrophic cardiomyopathy and the hearts of C57BL/6 mice underwent cardiac hypertrophy induced by transverse aortic constriction (TAC) surgery or isoproterenol (ISO) infusion. In neonatal rat cardiomyocytes (NRCMs), siRNA-mediated JMJD1A knockdown facilitated ISO or angiotensin II-induced increase in cardiomyocyte size, protein synthesis, and expression of hypertrophic fetal genes, including atrial natriuretic peptide (Anp), brain natriuretic peptide (Bnp), and Myh7. By contrast, overexpression of JMJD1A with adenovirus repressed the development of ISO-induced cardiomyocyte hypertrophy. We observed that JMJD1A reduced the production of total cellular and mitochondrial levels of reactive oxygen species (ROS), which was critically involved in the effects of JMJD1A because either N-acetylcysteine or MitoTEMPO treatment blocked the effects of JMJD1A deficiency on cardiomyocyte hypertrophy. Mechanism study demonstrated that JMJD1A promoted the expression and activity of Catalase under basal condition or oxidative stress. siRNA-mediated loss of Catalase blocked the protection of JMJD1A overexpression against ISO-induced cardiomyocyte hypertrophy. These findings demonstrated that JMJD1A loss promoted cardiomyocyte hypertrophy in a Catalase and ROS-dependent manner.
\end{abstract}

\section{Introduction}

Epigenetic regulation and posttranslational regulation of histone and nonhistone proteins are critically involved in the development of cardiac hypertrophy [1-3]. The histone deacetylases essentially participate in the development of cardiac hypertrophy by regulating the metabolism, mitochondrial homeostasis, and gene transcription [4-8]. In comparison to histone acetylation, the roles of histone methylation enzymes in cardiac hypertrophy are largely unknown. Lysine methylation is one of the most prominent histone posttranslational modifications that regulate chromatin structure and gene expression. Changes in histone lysine methylation status have been observed during cancer formation and development, which is a consequence of the dysregulation of histone lysine methyltransferases or demethylases $[9,10]$. Recent studies have implicated the roles of histone methylation/demethylation in cardiac hypertrophy and fibrosis $[10,11]$.

The JMJD (JmjC domain-containing) proteins family is composed of 30 members in humans based on the presence of the roughly 150 amino acid-long JmjC domain [12]. One of the largest JMJD subfamilies that has recently attracted much attention is the JMJD2 proteins (JMJD2AJMJD2D), which are capable of recognizing di- and trimethylated $\mathrm{H} 3 \mathrm{~K} 9$ and $\mathrm{H} 3 \mathrm{~K} 36$ as well as trimethylated $\mathrm{H} 1.4 \mathrm{~K} 26$ as substrates [9]. The most studied member of the JMJD2 family may be JMJD2A. A major study focusing on JMJD2A has been in transcription regulation, where it may either stimulate or repress gene transcription. JMJD2A functions in human Wiskott-Aldrich syndrome [13], Kaposi's sarcomaassociated herpesvirus replication [14], cardiac hypertrophy 
[15], and DNA repair [16]. For instance, JMJD2A promotes cardiac hypertrophy in response to hypertrophic stimuli in mice through binding to the FHL1 promoter, upregulating FHL1 expression, and downregulating $\mathrm{H} 3 \mathrm{~K} 9$ trimethylation [15].

JMJD1A is another member of this family. The roles of JMJD1A in tumor biology are widely studied. For instance, JMJD1A promotes alternative splicing of AR variant 7 (AR-V7) in prostate cancer cells [17]. JMJD1A regulates the transcriptional program of the androgen receptor in prostate cancer cells [18]. JMJD1A also promotes urinary bladder cancer progression by enhancing glycolysis through the coactivation of hypoxia-inducible factor $1 \alpha$ [19]. In addition, JMJD1A promotes cell growth and progression via transactivation of $\mathrm{c}-\mathrm{Myc}$ expression and predicts a poor prognosis in cervical cancer. JMJD1A was also reported to participate in thermogenesis [20]. Regulation of c-Myc expression by the histone demethylase JMJD1A is essential for prostate cancer cell growth and survival [21]. A previous study revealed the participation of JMJD1A in cardiac hypertrophy, but the underlying mechanisms are not fully understood [22]. In this study, we aimed at investigating the potential function and mechanism of JMJD1A in cardiac hypertrophy.

\section{Materials and Methods}

2.1. Patients. Human heart samples were obtained from the First Affiliated Hospital of Jiamusi University transplant program. Control samples were obtained intraoperatively from nonfailing hearts undergoing ventricular corrective surgery. Failing heart specimens were obtained from diseased hearts that were removed during orthotopic heart transplantation. Informed consent was obtained from all patients participating in this study. All procedures involving human tissue use were approved by the Ethics Review Board of the First Affiliated Hospital of Jiamusi University.

2.2. Experimental Animal Models of Cardiac Hypertrophy. 812 weeks old C57BL/6 mice were subjected to TAC surgery for 28 days to induce cardiac hypertrophy. The control mice were undergoing sham surgery. ISO (Sigma-Aldrich) was dissolved in $150 \mathrm{mM} \mathrm{NaCl}$ and $1 \mathrm{mM}$ acetic acid, and they were delivered $(8.7 \mathrm{mg} / \mathrm{kg} / \mathrm{d}$ for 28 days) by implanting of Osmotic Minipumps (model 2004; ALZET) into the abdomens of adult mice. Control mice underwent the same procedure, except that the respective pumps were filled only with vehicle (150 mM NaCl and $1 \mathrm{mM}$ acetic acid). The development of hypertrophy was judged noninvasively by the use of echocardiography. The animal study was approved by the Ethics Review Board of Animal Study at the First Affiliated Hospital of Jiamusi University.

2.3. Isolation and Culture of Neonatal Rat Cardiomyocytes. Neonatal rat cardiomyocytes (NRCMs) were cultured and infected with adenoviral vectors as described earlier [23]. The NRCMs were cultured in DMEM supplemented with $10 \%$ fetal bovine serum (Thermo Fisher), $0.1 \mathrm{mM} 5$ bromodeoxyuridine (to inhibit fibroblast proliferation), and $1 \%$ penicillin/streptomycin (Invitrogen). The animal study was approved by the Ethics Review Board of Animal Study at the First Affiliated Hospital of Jiamusi University.

2.4. Packaging of Adenovirus. Replication-defective adenoviral vectors expressing rat Jmjd1a (Ad-Jmjd1a) or control green fluorescent protein (Ad-Ctrl) were generated using the AdEasy Vector kit (Quantum Biotechnologies) according to previously described methods.

2.5. siRNA Transfection. For knocking down the expression of Jmjd1a or Catalase, siRNA was synthesized and transfected into NRCMs with the RNAiMax transfection kit (Invitrogen). The siRNA sequence was as follows. siJmjd1a-1\# $5^{\prime}$ GCACAGTCCTCCATACGTT-3', siJmjd1a-2\# 5' - GGAU GUAAACAGUCUUCGA-3', siCatalase: $5^{\prime}$ - CCAGAU ACUCCAAGGCAAATT-3'.

2.6. Western Blot. Total protein was extracted from NRCMs or heart tissues with RIPA and protease inhibitors. Then, the protein was subjected to western blot as described previously [24]. The following antibodies were used: anti-JMJD1A antibody (Abcam, \#ab106456), anti- $\beta$-Actin antibody (Cell Signaling Technology, \#4967), anti-Catalase antibody (Santa Cruz, \#sc-271803), anti-H3K9me1 antibody (Abcam, \#ab9045), anti-H3K9me2 antibody (Abcam, \#ab1220), and anti-Histone $\mathrm{H} 3$ antibody (Abcam, \#ab8898).

2.7. Quantitative Real-Time PCR ( $q R T-P C R)$. Total cellular RNA was isolated from cardiomyocytes or heart tissues using the TRIzol Reagent (Invitrogen). One microgram of DNase-I-treated RNA was reverse transcribed using the SuperScript III kit (Invitrogen). The resultant cDNA was subjected to quantitative real-time PCR with SYBR GREEN II (TAKARA). The primers used for qRT-PCR are listed in the Supplementary data (available here).

2.8. Induction of Cardiomyocyte Hypertrophy. NRCMs were cultured for 48 hours, then infected with indicated adenovirus or transfected with siRNA and cultured in serumfree medium for 24 hours. Next, the cells were treated with ISO $(1 \mu \mathrm{M})$ or angiotensin II (Sigma, \#A9525, $1 \mu \mathrm{M})$ for 48 hours in serum-free medium to induce cardiomyocyte hypertrophy.

2.9. Cardiomyocyte Size Analysis. Then, cardiomyocytes were stained with $\alpha$-actinin antibody (Siamg, \#A7811). The cell size of cardiomyocytes was measured by using NIH ImageJ software (http://rsbweb.nih.gov/ij/). More than 100 cells were quantified per experiment, and the average values of three independent experiments were used for quantification.

2.10. Protein Synthesis Analysis. The hypertrophic response of cells was measured by $\left[{ }^{3} \mathrm{H}\right]$-leucine incorporation into total protein as described previously [25].

2.11. Measurement of Total and Mitochondrial ROS Level. Total ROS and mitochondrial ROS levels were measured with the DHE staining kit or mitoSOX staining kit prospectively as described previously [8]. The relative ROS levels were monitored and evaluated with image J. 
2.12. Catalase Activity Assay. The activity of Catalase in cardiomyocytes was monitored with Catalase Activity Assay Kit (Abcam, \# ab83464).

2.13. Statistical Analysis. All values are expressed as the mean \pm S.D. Statistical differences among groups were determined using either Student's $t$ test (for two groups) or oneway ANOVA (for more than two groups) using Graph-Pad Prism 8 Software. $p$ values $<0.05$ were considered significant.

\section{Results}

3.1. JMJD1A Expression Is Reduced during Cardiac Hypertrophy. The roles of JMJD1A in the cardiovascular system remain unknown; here, we aimed at investigating the potential roles of JMJD1A in cardiac hypertrophy. We first analyzed the expression of JMJD1A in hypertrophic hearts from patients and mice. We collected heart tissues from patients with hypertrophic cardiomyopathy (HCM, $n=10$ ) and control healthy donors $(n=5)$. The mRNA levels of hypertrophic fetal genes were significantly upregulated in the hearts of HCM compared with the control donors (Figure 1(a)). We also analyzed the expression of JMJD1A in these samples and found that the mRNA level of JMJD1A was significantly downregulated in hypertrophic hearts of human (Figure 1(b)). In addition, we performed western blot to examine the protein level of JMJD1A. The results showed that the protein level of JMJD1A was significantly downregulated in the hearts of patients with HCM (Figure 1(c)). JMJD1A is a histone demethylase of $\mathrm{H} 3 \mathrm{~K} 9 \mathrm{me} 1 / 2$. Indeed, we observed an increase in $\mathrm{H} 3 \mathrm{~K} 9 \mathrm{mel}$ and $\mathrm{H} 3 \mathrm{~K} 9 \mathrm{me} 2$ in the HCM group (Figure 1(c)). Next, we analyzed the expression of Jmjd1a in mice with experimental cardiac hypertrophy. Cardiac hypertrophy was induced in C57BL/6 mice with TAC surgery. The expression of hypertrophic fetal genes was analyzed at four weeks post-TAC surgery. The results showed that the mRNA levels of hypertrophic fetal genes were remarkedly upregulated in TAC-induced hypertrophic hearts (Figure 1(d)). We next tested the expression of Jmjdla mRNA and protein levels and found that either the mRNA or protein level of Jmjdla was significantly downregulated in TAC-induced hypertrophic hearts (Figures 1(e) and 1(f)), which was accomplished with the increase in $\mathrm{H} 3 \mathrm{~K} 9 \mathrm{mel}$ and $\mathrm{H} 3 \mathrm{~K} 9 \mathrm{me} 2$ (Figure 1(f)). In addition, we also induced cardiac hypertrophy in mice by infusing of ISO for four weeks. The ISO infusion significantly upregulated the expression of hypertrophic fetal genes in the hearts of mice (Figure $1(\mathrm{~g})$ ). Importantly, the results showed that ISO infusion downregulated the mRNA and protein levels of Jmjd1a in the hearts of mice infused with ISO for four weeks (Figures 1(h) and 1(i)), in couple with the upregulation of $\mathrm{H} 3 \mathrm{~K} 9 \mathrm{mel}$ and $\mathrm{H} 3 \mathrm{~K} 9 \mathrm{me} 2$ (Figure 1(i)). Taken together, these findings demonstrated that the expression of JMJD1A was downregulated whereas $\mathrm{H} 3 \mathrm{~K} 9 \mathrm{me} 1$ and $\mathrm{H} 3 \mathrm{~K} 9 \mathrm{me} 2$ were increased in hypertrophic hearts of humans and mice.

3.2. JM9JD1A Represses the Development of Cardiomyocyte Hypertrophy. Next, we investigated whether the downregula- tion of JMJD1A during cardiac hypertrophy contributes to the development of cardiomyocyte hypertrophy. We isolated cardiomyocytes from the hearts of neonatal rats. Then, we knockdown the expression of Jmjd1a in neonatal rat cardiomyocytes (NRCMs) by transfecting the cells with siRNA (Figure 2(a)) and treated with the cells with ISO to induce cardiomyocyte hypertrophy. The results showed that ISO treatment significantly increased the size of NRCMs and Jmjd1a knockdown promoted the effects on ISO-induced increase in cardiomyocyte size (Figure 2(b)). Protein synthesis is a hallmark of cardiomyocyte hypertrophy. Indeed, ISO treatment induced the $\left[{ }^{3} \mathrm{H}\right]$-leucine incorporation into cardiomyocytes and Jmjdla knockdown promoted the effects of ISO (Figure 2(c)). We also tested the expression of hypertrophic fetal genes and found that Jmjd1a knockdown facilitated the expression of hypertrophic fetal genes (Anp, Bnp, and Myh7) induced by ISO (Figure 2(d)). We also induced cardiomyocyte hypertrophy with angiotensin II (Ang II). Similar results were obtained as evidenced by cardiomyocyte size and hypertrophic gene expression (Figures 2(e) and 2(f)).

Next, we investigated whether JMJD1A overexpression could repress the development of cardiomyocyte hypertrophy. We overexpressed JMJD1A in NRCMs by infecting the cells with adenovirus carrying Jmjd1a (Ad-Jmjd1a, Figure 3(a)). We then treated the cells with ISO and analyzed the hypertrophic phenotype of cardiomyocytes. The results showed that JMJD1A overexpression repressed the effects of ISO-induced increase in cardiomyocyte size and protein synthesis (Figures 3(b) and 3(c)). Finally, we analyzed the effects of JMJD1A overexpression on ISO-induced expression of hypertrophic fetal genes and found that JMJD1A overexpression inhibited the expression of hypertrophic fetal genes (Figure 3(d)). Collectively, these findings demonstrated that JMJD1A inhibited cardiomyocyte hypertrophy.

3.3. ROS Is Involved in the Function of JMJD1A during Cardiomyocyte Hypertrophy. Elevated total and mitochondrial ROS levels contribute to the development of cardiac hypertrophy [5]. To explore the potential mechanism underlying JMJD1A-mediated functions during cardiomyocyte hypertrophy, we tested the effects of JMJD1A on total cellular and mitochondrial ROS. Upon ISO treatment, the levels of total cellular and mitochondrial ROS were upregulated, which was facilitated by knocking down the expression of Jmjdla with siRNA in NRCMs (Figure 4(a)). By contrast, adenovirus-mediated Jmjd1a overexpression repressed both total cellular and mitochondrial ROS levels (Figure 4(b)). Next, we examined whether the effects of JMJD1A on ROS contributed to its function during cardiomyocyte hypertrophy. Therefore, we used N-acetylcysteine (NAC) and MitoTEMPO to repress total cellular and mitochondrial ROS, respectively, which completely blocked the effects of Jmjd1a knockdown on ROS production (Figure 4(c)). We also observed that either NAC or MitoTEMPO treatment repressed cardiomyocyte hypertrophy and blocked the function of Jmjd1a knockdown as evidenced by cardiomyocyte size and the expression of hypertrophic fetal genes (Figures 4(d)-4(f)). Therefore, JMJD1A repressed the 


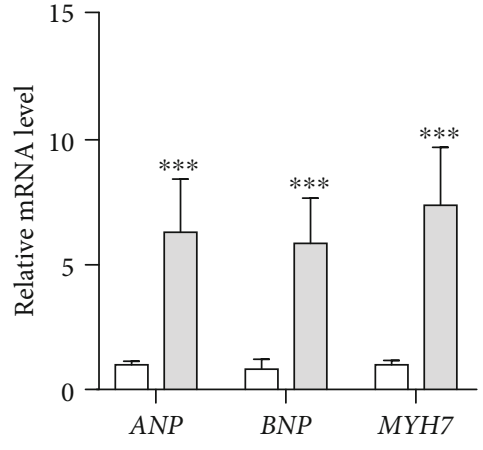

$\square$ Healthy

$\square$ HCM

(a)

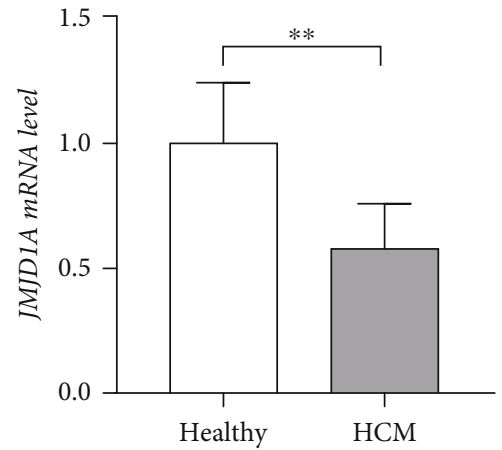

(b)

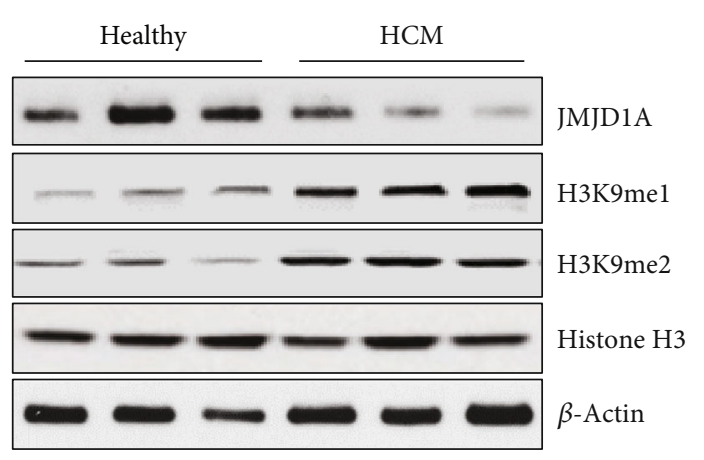

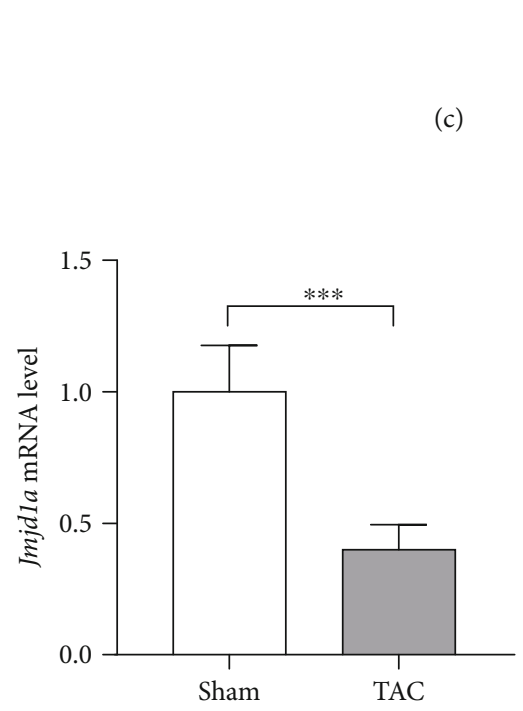

(e)

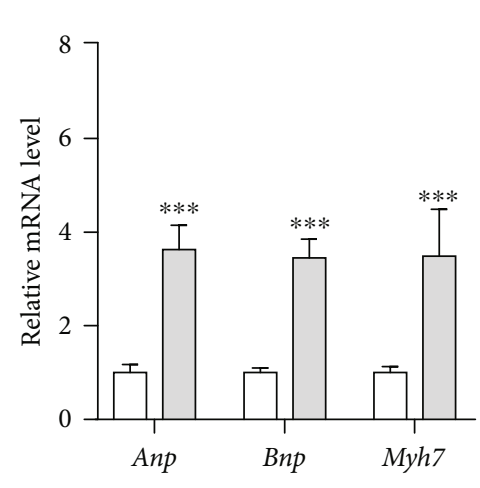

$\square$ Sham

(d)

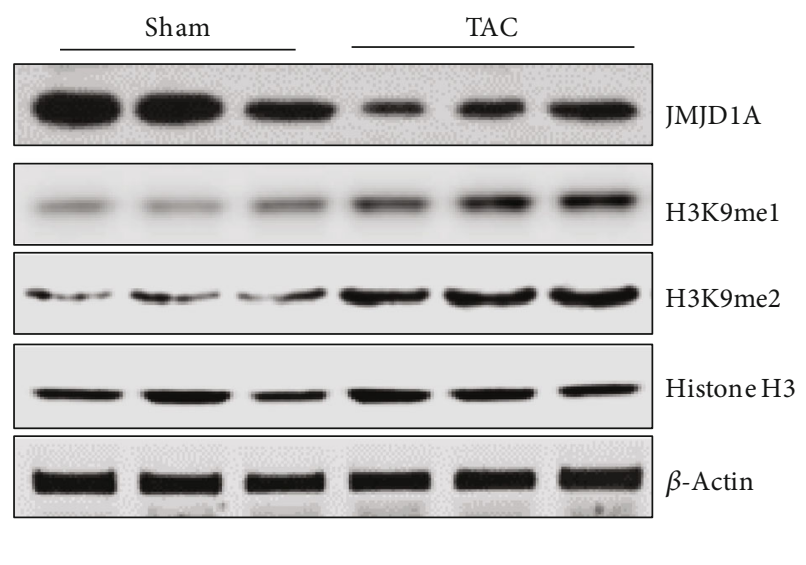

(f)

Figure 1: Continued. 


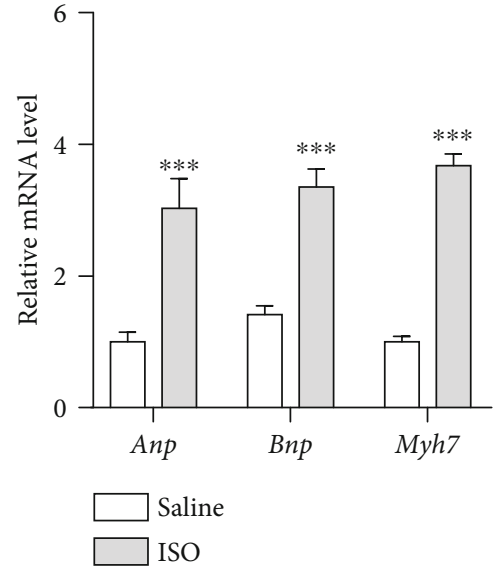

(g)

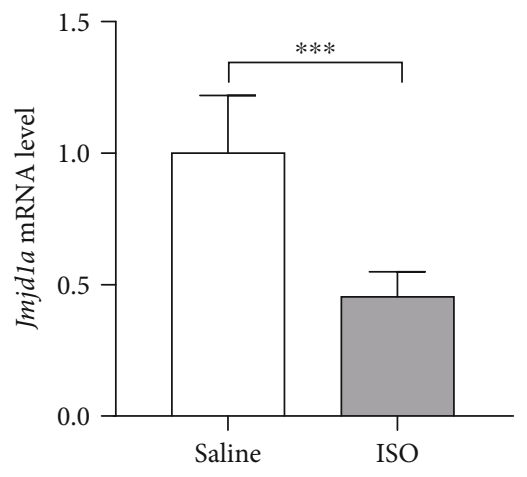

(h)

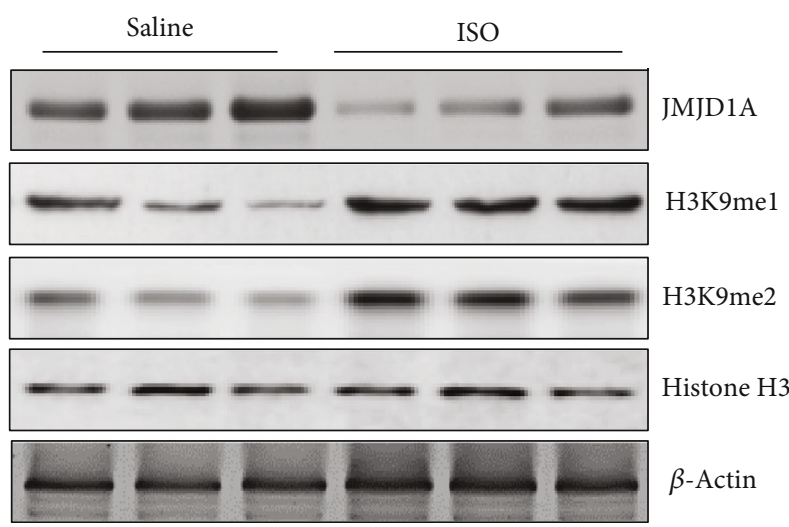

(i)

FIGURE 1: JMJD1A expression is downregulated during cardiac hypertrophy. (a) mRNA levels of hypertrophic fetal genes in the hearts from control or hypertrophic cardiomyopathy (HCM). ANP: atrial natriuretic peptide; BNP: brain natriuretic peptide; MYH7: beta-myosin heavy chain. $n=5$ in each group, ${ }^{* * *} p<0.001 v s$. healthy group. (b) mRNA levels of JMJD1A in the hearts from control or hypertrophic cardiomyopathy (HCM). $n=5$ in control group, $n=10$ in HCM group, ${ }^{* *} p<0.01$. (c) Protein levels of JMJD1A and H3K9me1/2 in the hearts from control or hypertrophic cardiomyopathy (HCM). (d) mRNA levels of hypertrophic fetal genes in the hearts of mice underwent sham or TAC surgery. TAC: transverse aortic constriction. $n=5$ in each group, ${ }^{* * *} p<0.001$ vs. sham group. (e) mRNA levels of Jmjd1a in the hearts of mice underwent sham or TAC surgery. $n=5$ in each group, ${ }^{* * *} p<0.001 v s$. sham group. (f) Protein levels of JMJD1A and $\mathrm{H} 3 \mathrm{~K} 9 \mathrm{me1} / 2$ in the hearts from mice underwent sham or TAC surgery for four weeks. (g) mRNA levels of hypertrophic fetal genes in the hearts of mice underwent saline or ISO infusion for four weeks. $n=5$ in each group, ${ }^{* * *} p<0.001 v s$. saline group. (h) mRNA levels of Jmjd1a in the hearts of mice underwent saline or ISO infusion for four weeks. $n=5$ in each group, ${ }^{* * *} p<0.001 v s$. saline group. (i) Protein levels of JMJD1A and H3K9me1/2in the hearts from mice underwent saline or ISO infusion for four weeks.

production of ROS, which was critically involved in the function of JMJD1A in cardiomyocyte hypertrophy.

3.4. JMJD1A Promotes the Expression of Catalase to Regulate ROS and Cardiomyocyte Hypertrophy. We next investigated the mechanism underlying JMJD1A function in the regulation of ROS. We knocked down the expression of Jmjd1a in NRCMs and tested the enzymes in regulating cellular and mitochondrial ROS levels, including Sod1, Sod2, Catalase, $p 66^{\text {shc }}$, Trx, Grx, and Nrf2. The results showed that only the expression of Catalase was repressed by Jmjd1a knockdown, whereas the expression of the other factors was not affected by Jmjd1a (Figures 5(a) and 5(b)). To confirm the effect of Jmjd1a on Catalase expression, we overexpressed Jmjd1a in NRCMs and observed that Jmjdla overexpression promoted the expression of Catalase under basal condition or under the stress induced by ISO (Figure 5(c)). In addition, we observed that Jmjd1a knockdown reduced catalase activity, whereas Jmjd1a overexpression led to oppose effects (Figure 5(d)), indicating that JMJD1A controlled Catalase expression and activity. Next, we investigated whether Catalase was critically involved in the development of cardiomyocyte hypertrophy. We knocked down the expression of Catalase with siRNA in NRCMs (Figure 5(e)). Catalase knockdown promoted the ISO-induced increase in cardiomyocyte size, protein synthesis, and expression of hypertrophic fetal genes. Interestingly, we observed that Jmjd1a overexpression was unable to regulate cardiomyocyte hypertrophy in NRCMs with Catalase deficiency (Figures 5(f) and 5(h)). These findings revealed that Catalase contributed to the function of JMJD1A in cardiomyocyte hypertrophy. 


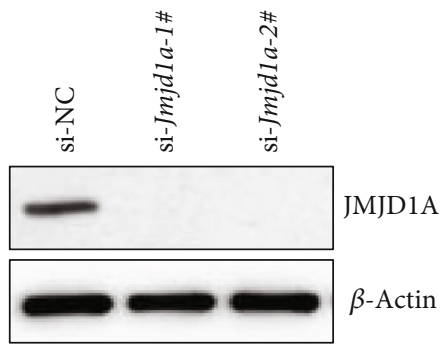

(a)

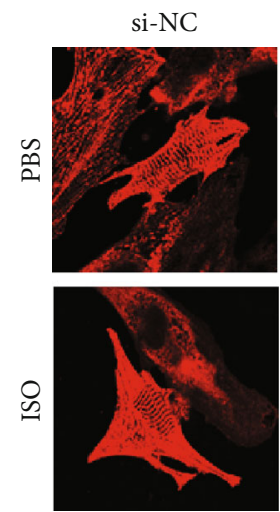

si-Jmjd1a-1\#

si-Jmjd1a-2\#
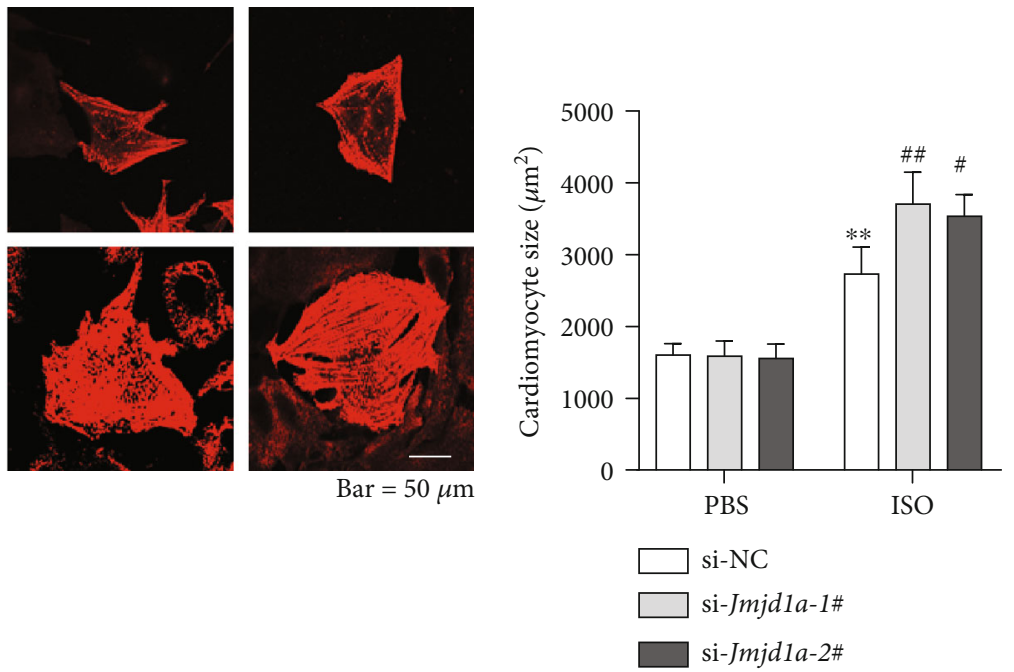

(b)

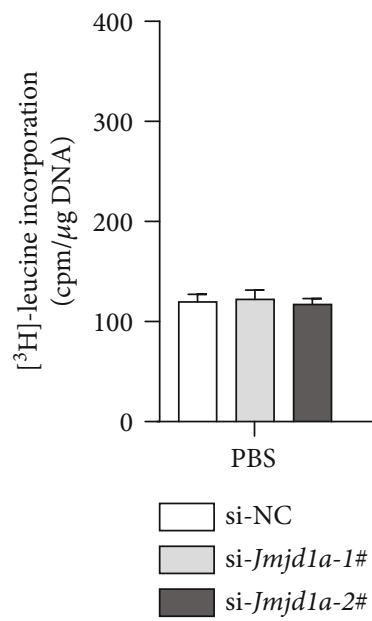

(c)

Figure 2: Continued. 

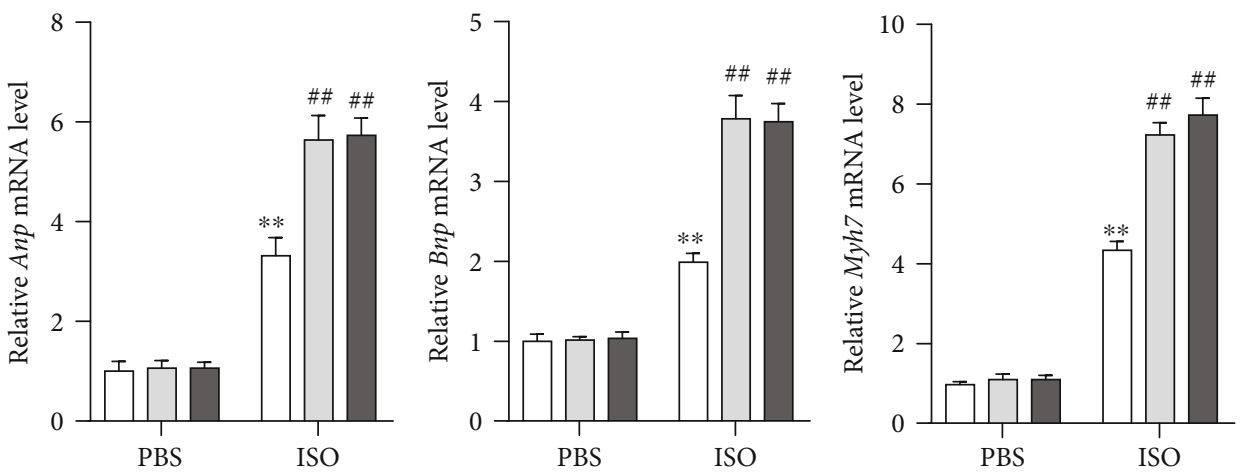

\section{si-NC \\ si-Jmjd1a-1\# \\ si-Jmjd1a-2\#}

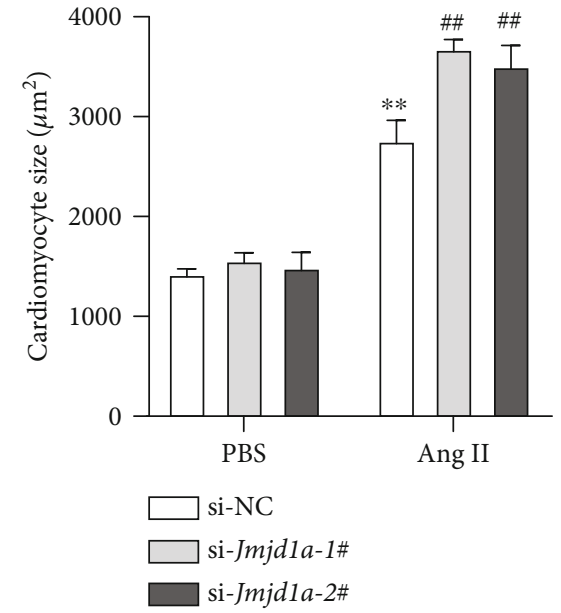

(e)

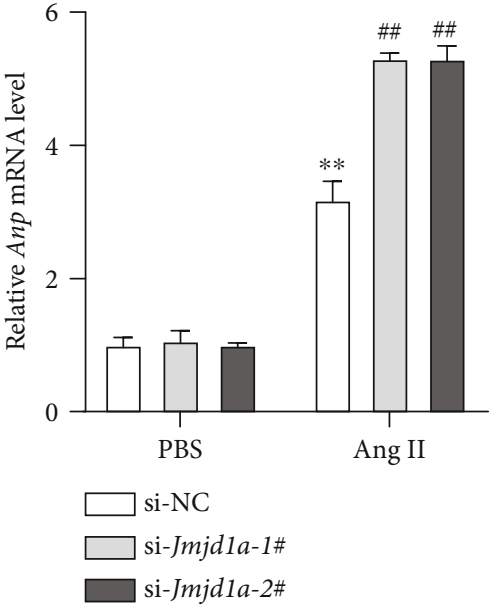

(f)

FIGURE 2: JMJD1A knockdown promotes cardiomyocyte hypertrophy of neonatal rat cardiomyocytes. (a) Representative western blot showing the expression of JMJD1A protein in neonatal rat cardiomyocytes transfected with si-Jmjd1a or si-NC. NRCMs were transfected with si-NC or si-Jmjd1a for 36 hours. (b) Jmjd1a knockdown promotes an ISO-induced increase in cardiomyocyte size. Representative images and quantitative results are shown. NRCMs were transfected with si-NC or si-Jmjd1a for 24 hours and then treated with ISO $(1 \mu \mathrm{M})$ for 48 hours. ${ }^{* *} p<0.01$ vs. PBS+si-NC, $\# p<0.05$ and ${ }^{\# \#} p<0.01$ vs. ISO+si-NC. (c) Jmjd1a knockdown promotes ISO-induced protein synthesis in cardiomyocytes. NRCMs were transfected with si-NC or si-Jmjd1a for 24 hours and then treated with ISO ( $1 \mu \mathrm{M})$ for 48 hours. ${ }^{* *} p<0.01$ vs. PBS+si-NC, ${ }^{\# \#} p<0.01$ vs. ISO+si-NC. (d) Jmjdla knockdown promotes ISO-induced expression of hypertrophic fetal genes. NRCMs were transfected with si-NC or si-Jmjd1a for 24 hours and then treated with ISO $(1 \mu \mathrm{M})$ for 48 hours. ${ }^{* *} p<0.01 v s$. PBS+si-NC, ${ }^{\# \#} p<0.01$ vs. ISO+si-NC. (e) Jmjd1a knockdown promotes angiotensin II (Ang II)-induced increase in cardiomyocyte size. Representative images and quantitative results are shown. NRCMs were transfected with si-NC or si-Jmjd1a for 24 hours and then treated with Ang II $(1 \mu \mathrm{M})$ for 48 hours. ${ }^{* *} p<0.01 v s$. PBS+si-NC, ${ }^{\# \#} p<0.01 v s$. Ang II+si-NC. (f) Jmjd1a knockdown promotes Ang II-induced expression of hypertrophic fetal genes. NRCMs were transfected with si-NC or si-Jmjd1a for 24 hours and then treated with Ang II $(1 \mu \mathrm{M})$ for 48 hours. ${ }^{* *} p<0.01$ vs. PBS + si-NC, ${ }^{\# \#} p<0.01 v s$. Ang II+si-NC.

\section{Discussion}

Histone H3K9 demethylase JMJD1A is a signal-sensing scaffold that regulates acute chromatin dynamics via SWI/SNF association for thermogenesis [20]. JMJD1A demethylates $\mathrm{H} 3 \mathrm{~K} 9 \mathrm{me} 2$ at the promoter regions of Tcl1, Tcfcp 2l1, and Zfp57 and positively regulates the expression of these pluripotency-associated genes [26]. The hypoxia-inducible epigenetic regulators JMJD1A and G9a provide a mechanistic link between angiogenesis and tumor growth [27]. In addition, JMJD1A modulates hepatic stellate cells activation and liver fibrosis by epigenetically regulating peroxisome proliferator-activated receptor $\gamma$ [28]. JMJD1A interacts with the Myocardin factors to regulate smooth muscle cell differentiation marker gene expression [29]. Although a previous study has revealed the potential participation of JMJD1A in cardiac hypertrophy [22], the roles and underlying mechanisms of JMJD1A in cardiac hypertrophy across species are not fully understood. Here, we showed that JMJ1A participated in cardiac hypertrophy via targeting catalase to 


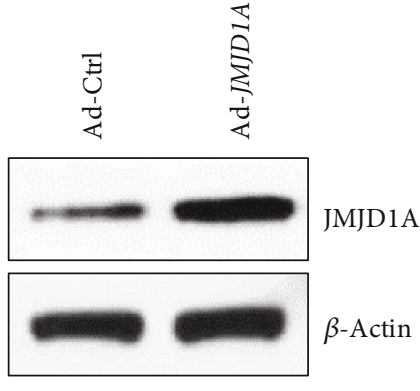

(a)
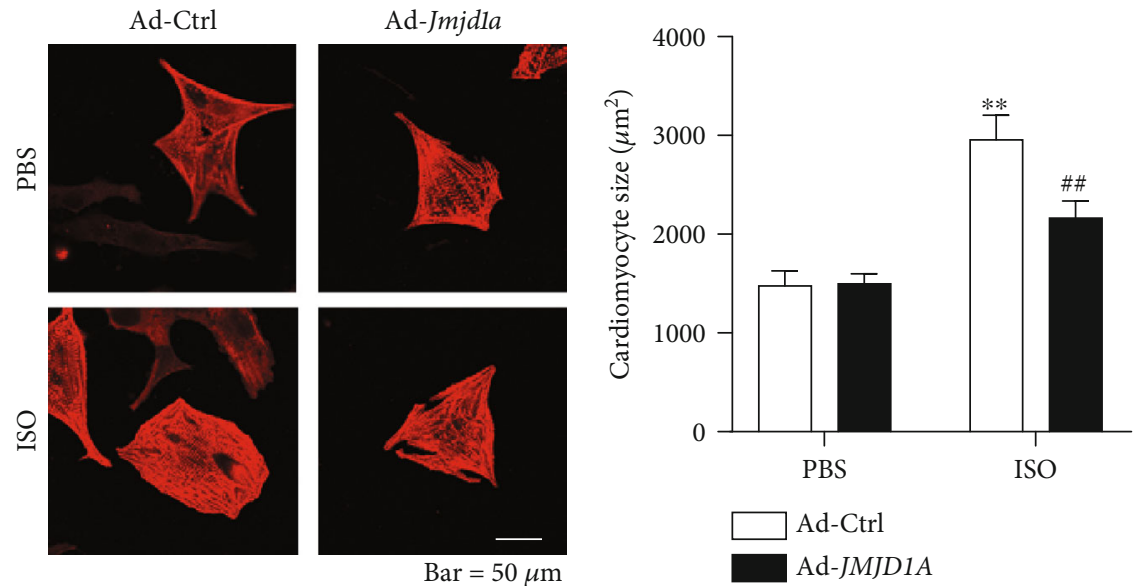

(b)

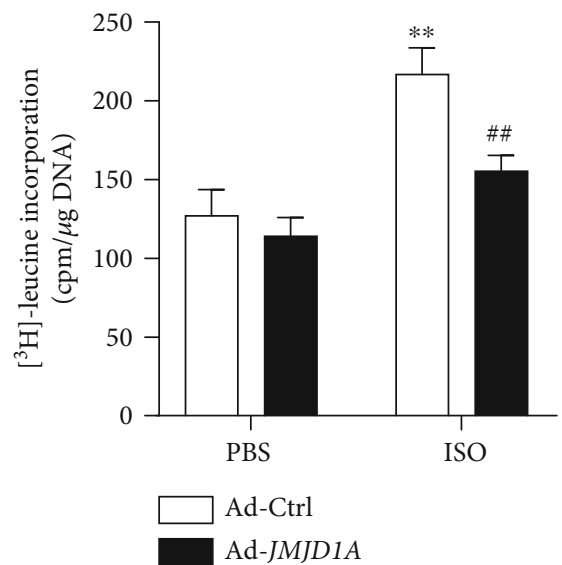

(c)
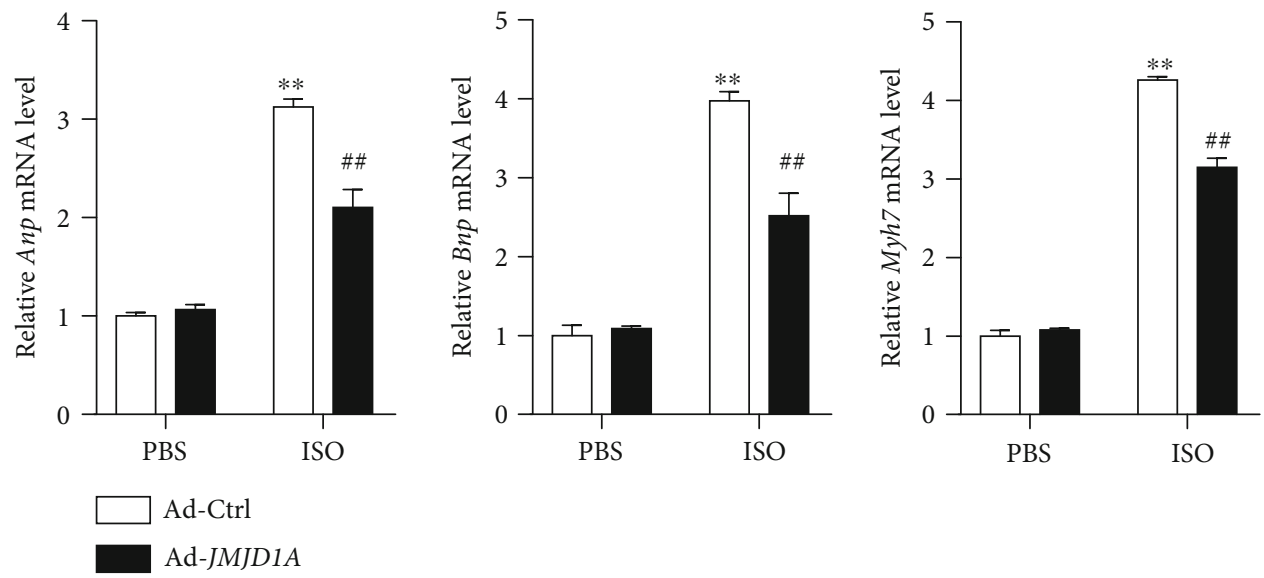

(d)

FIGURE 3: JMJD1A overexpression represses cardiomyocyte hypertrophy. (a) Representative western blot showing the expression of JMJD1A proteins in NRCMs infected with adenovirus carrying Jmjd1a or control adenovirus for 36 hours. (b) JMJD1A overexpression represses the ISO-induced increase in cardiomyocyte size. Representative images and quantitative results are shown. NRCMs were infected with indicated adenovirus for 24 hours and then treated with ISO $(1 \mu \mathrm{M})$ for 48 hours. ${ }^{* *} p<0.01 v s$. PBS+Ad-Ctrl, ${ }^{\# \#} p<0.01 v s$. ISO+Ad-Ctrl. (c) JMJD1A overexpression represses ISO-induced protein synthesis in cardiomyocytes. NRCMs were infected with indicated adenovirus for 24 hours and then treated with ISO $(1 \mu \mathrm{M})$ for 48 hours. ${ }^{* *} p<0.01 v s$. PBS+Ad-Ctrl, ${ }^{\# \#} p<0.01 v s$. ISO+Ad-Ctrl. (d) JMJD1A overexpression represses ISO-induced expression of hypertrophic fetal genes. NRCMs were infected with indicated adenovirus for 24 hours and then treated with ISO $(1 \mu \mathrm{M})$ for 48 hours. ${ }^{* *} p<0.01 v s$. PBS+Ad-Ctrl, ${ }^{\# \#} p<0.01 v s$. ISO+Ad-Ctrl. 

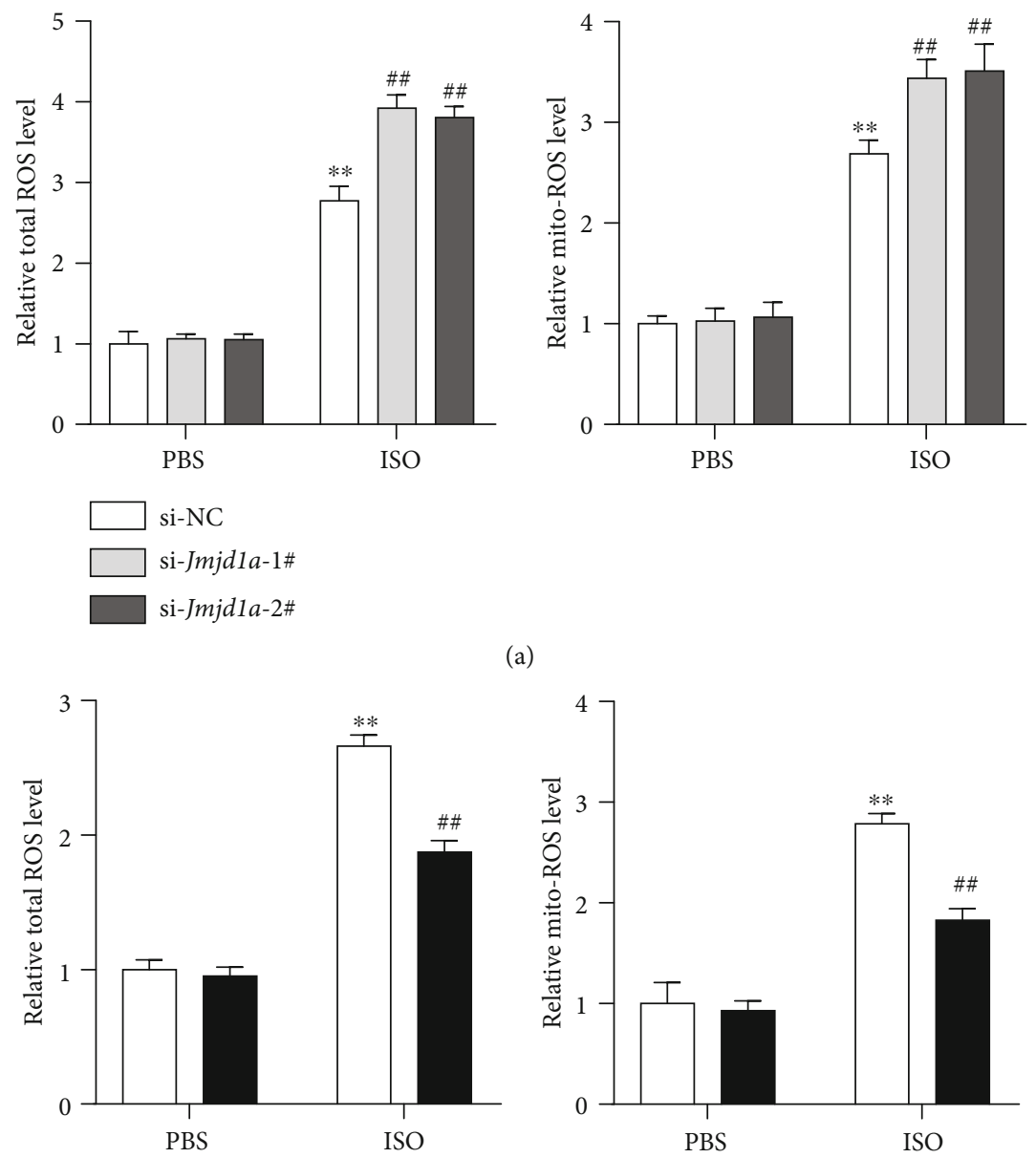

Ad-Ctrl

Ad-JMJD1A

(b)
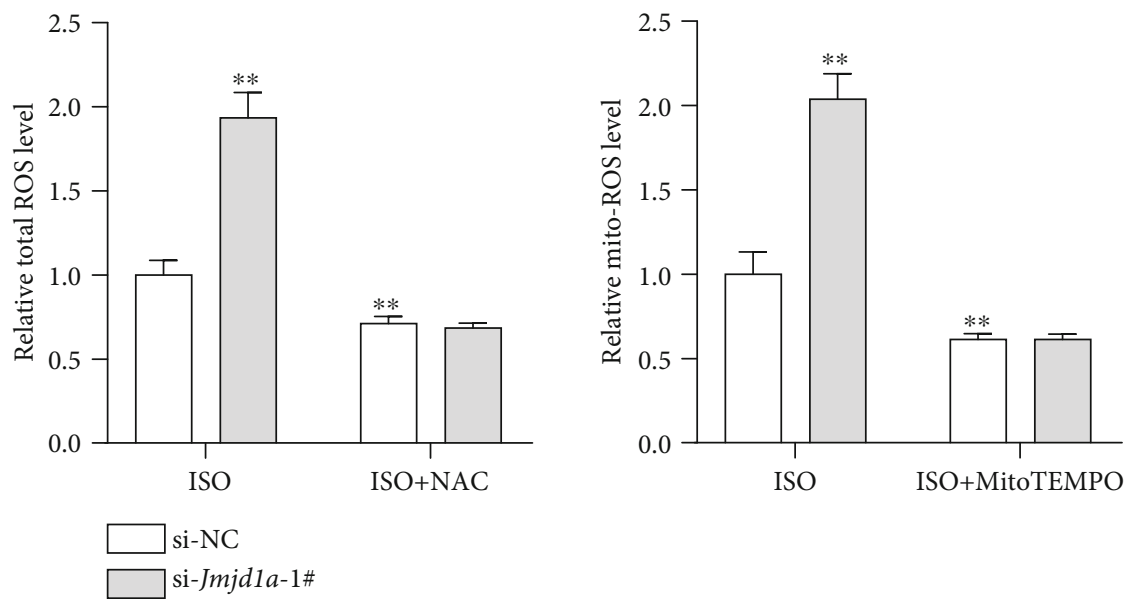

(c)

Figure 4: Continued. 

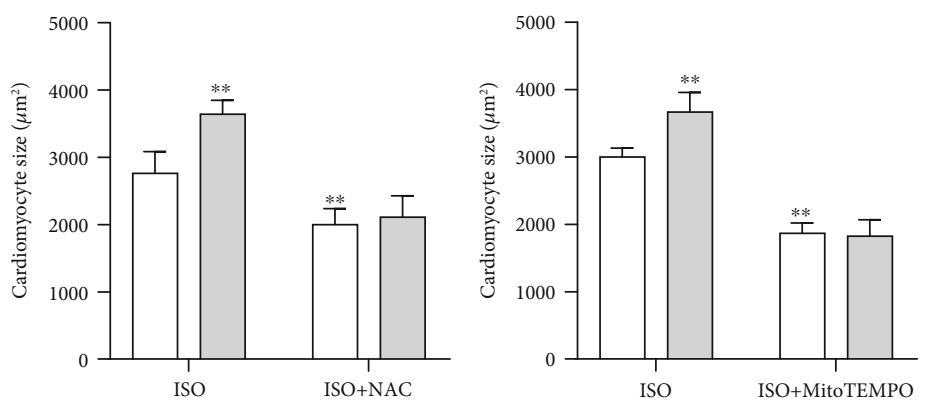

(d)
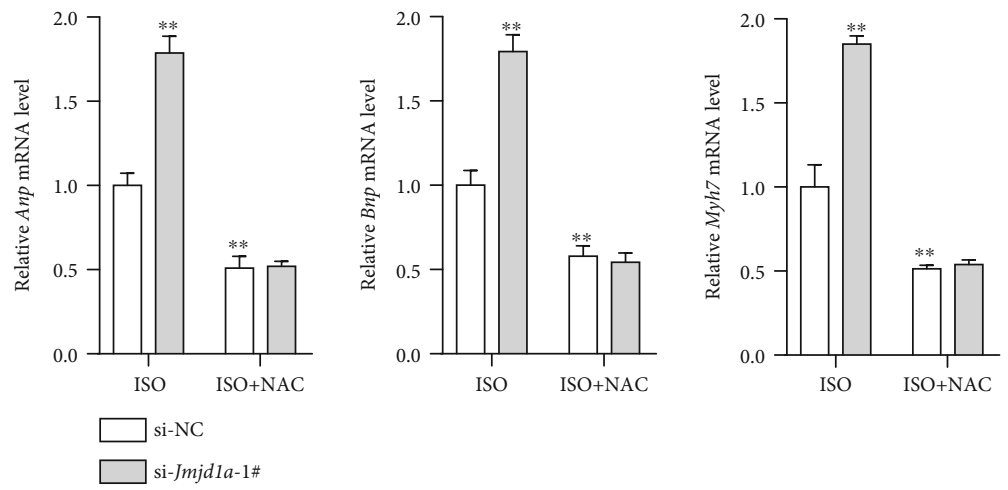

(e)
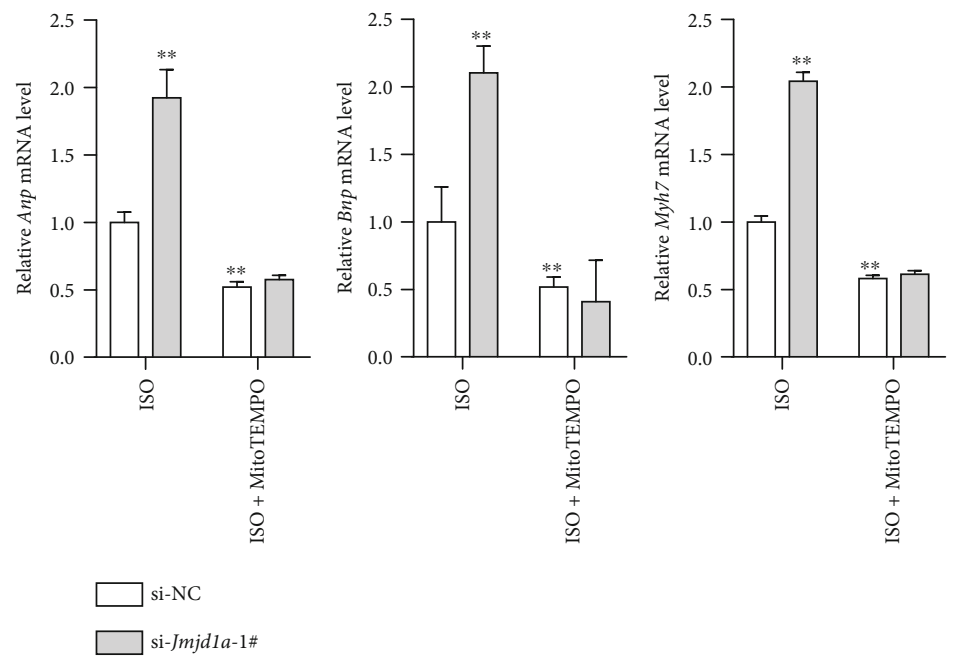

(f)

FIGURE 4: ROS contributes to the roles of JMJD1A in cardiomyocytes. (a) Jmjd1a knockdown promotes ISO-induced total cellular and mitochondrial ROS. NRCMs were infected with indicated adenovirus for 24 hours and then treated with ISO $(1 \mu \mathrm{M})$ for 12 hours. ${ }^{* *} p<$ 0.01 vs. PBS+si-NC and ${ }^{\# \#} p<0.01$ vs. ISO+si-NC. (b) JMJD1A overexpression reduces ISO-induced total cellular and mitochondrial ROS. NRCMs were infected with indicated adenovirus for 24 hours and then treated with ISO $(1 \mu \mathrm{M})$ for 12 hours. ${ }^{* *} p<0.01 v s$. PBS+si-NC and ${ }^{\# \#} p<0.01 v s$. ISO+si-NC. (c) NAC and MitoTEMPO repress Jmjd1a knockdown-mediated increase in total and mitochondrial ROS, respectively. NRCMs were transfected with indicated siRNA for 24 hours and then treated with ISO ( $1 \mu \mathrm{M})$ in the presence of NAC $(2 \mathrm{mM})$ or MitoTEMPO $(50 \mathrm{nM})$ for 12 hours. ${ }^{* *} p<0.01 v s$. PBS+si-NC and ${ }^{\# \#} p<0.01 v s$. ISO+si-NC. (d) NAC and MitoTEMPO repress Jmjdla knockdown-mediated increase in cardiomyocyte size. NRCMs were transfected with indicated siRNA for 24 hours and then treated with ISO $(1 \mu \mathrm{M})$ in the presence of NAC $(2 \mathrm{mM})$ or MitoTEMPO $(50 \mathrm{nM})$ for 48 hours. ${ }^{* *} p<0.01 v s$. PBS $+s i-\mathrm{NC}$. (e) NAC represses JMJD1A knockdown-mediated increase in expression of hypertrophic fetal genes. NRCMs were transfected with indicated siRNA for 24 hours and then treated with ISO $(1 \mu \mathrm{M})$ in the presence of NAC $(2 \mathrm{mM})$ for 48 hours. ${ }^{* *} p<0.01 v s$. PBS+si-NC. (f) MitoTEMPO represses the JMJD1A knockdown-mediated increase in the expression of hypertrophic fetal genes. NRCMs were transfected with indicated siRNA for 24 hours and then treated with ISO $(1 \mu \mathrm{M})$ in the presence of MitoTEMPO $(50 \mathrm{nM})$ for 48 hours. ${ }^{* *} p<0.01 v s$. PBS+si-NC. 


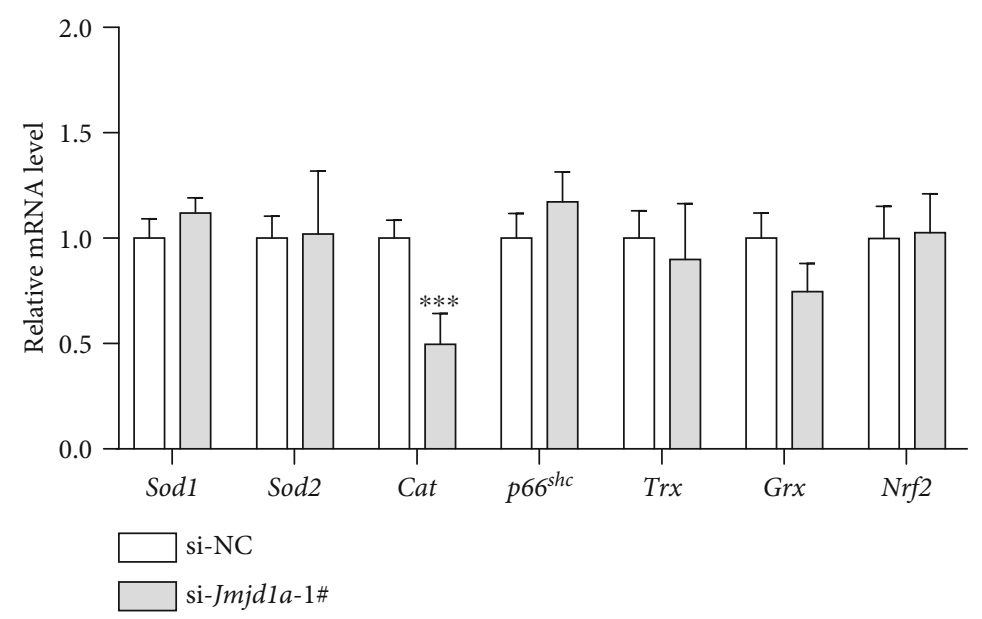

(a)

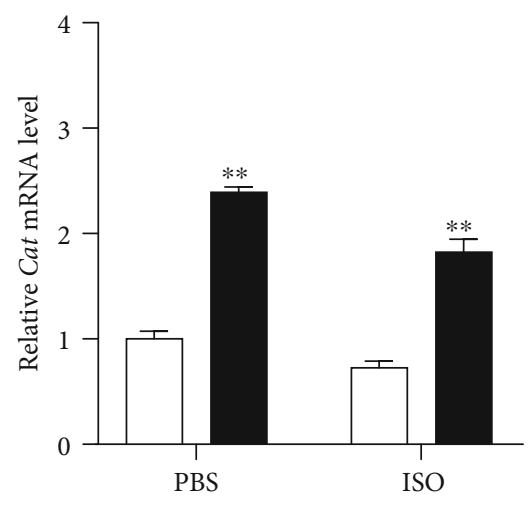

$\square$ Ad-Ctrl

Ad-JMJD1A

(c)

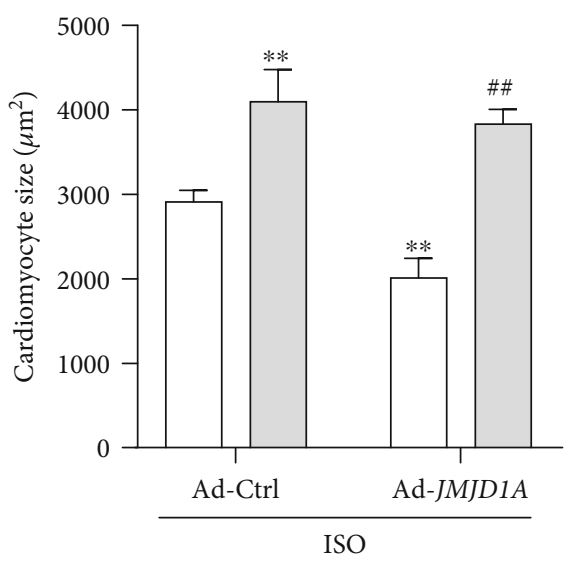

$\square$ si-NC si-Catalase

(f)

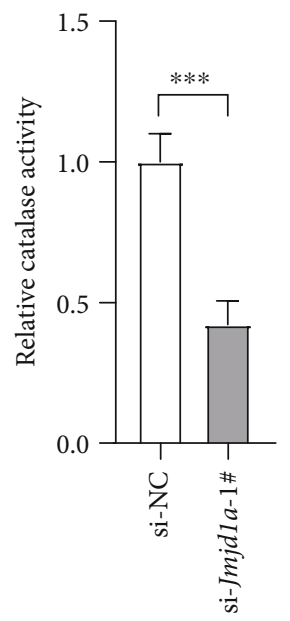

(d)

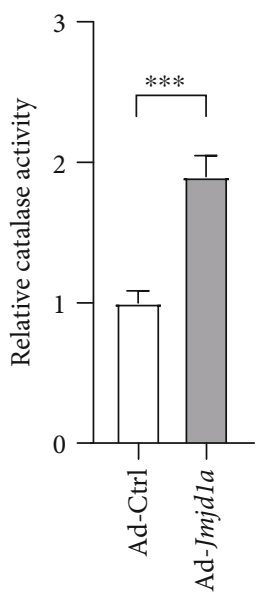

si-NC

si-Catalase

(g)

(e)

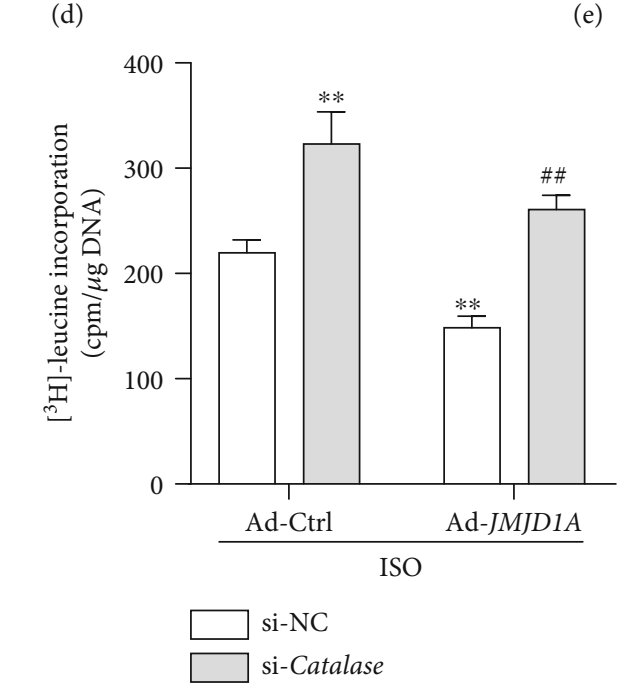

(b)

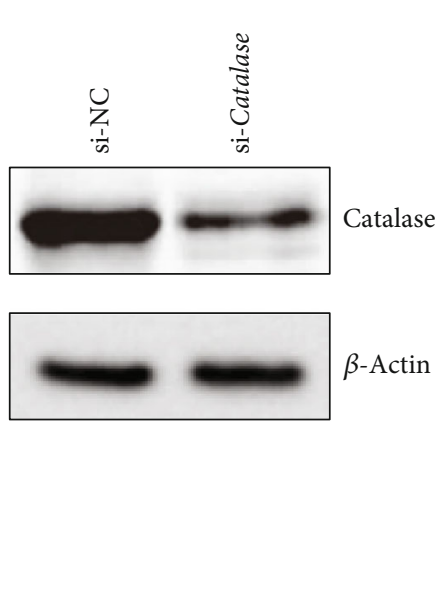

Figure 5: Continued. 


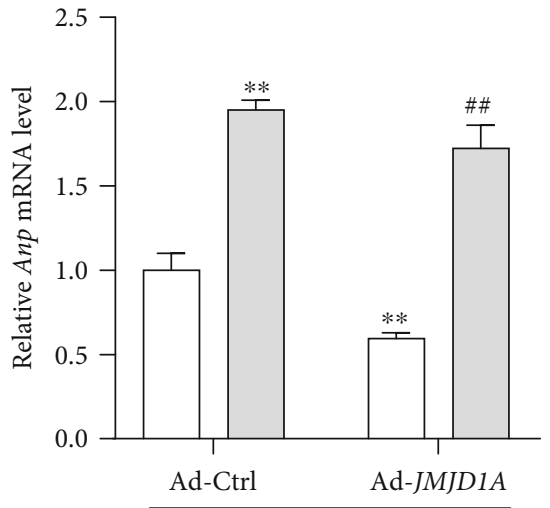

ISO

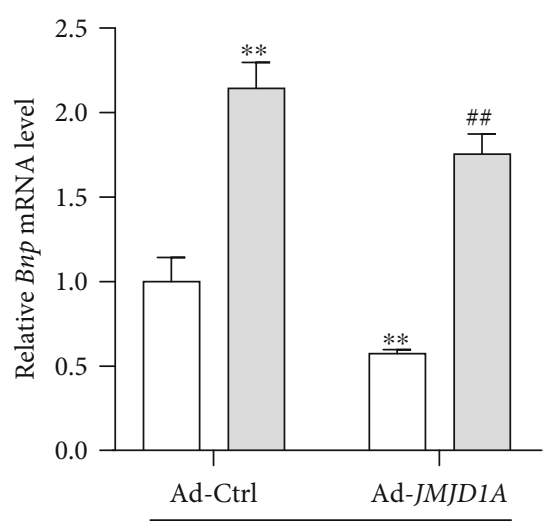

ISO

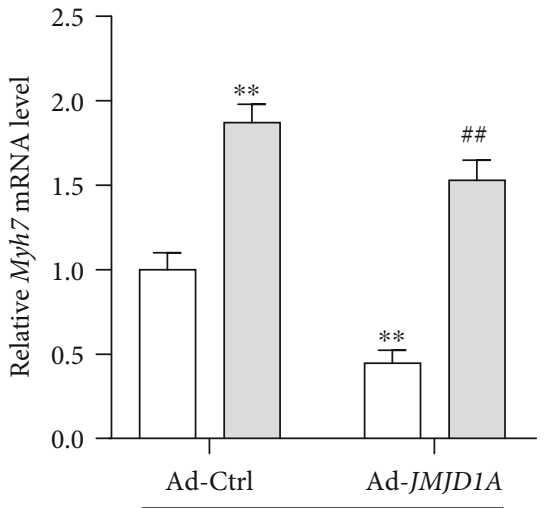

ISO

si-NC

si-Catalase

(h)

FIGURE 5: JMJD1A regulates catalase to modulate ROS and cardiomyocyte hypertrophy. (a) mRNA levels of oxidative genes and antioxidants in cardiomyocytes transfected with/without si-Jmjd1a. NRCMs were transfected with indicated siRNA for 36 hours. ${ }^{* * *} p<0.001 v s$. si-NC. (b) Protein levels of Catalase in cardiomyocytes transfected with/without si-Jmjd1a. NRCMs were transfected with indicated siRNA for 36 hours. (c) JMJD1A overexpression promotes the expression of Catalase under basal and ISO-induced stress. NRCMs were infected with indicated adenovirus for 24 hours and then treated with ISO $(1 \mu \mathrm{M})$ for an additional 24 hours. ${ }^{* *} p<0.01 v s$. Ad-Ctrl. (d) JMJD1A controls Catalase activity. Cardiomyocytes were treated as in (a) or (c), then, catalase activity in cardiomyocytes was analyzed with a kit. (e) Representative western blot results showing Catalase knockdown in cardiomyocytes. NRCMs were transfected with indicated siRNA for 36 hours. (f) Catalase knockdown blocked JMJD1A-mediated repression in cardiomyocyte hypertrophy. NRCMs were infected with indicated adenovirus for 24 hours and then transfected with si-NC or si-Catalase for 24 hours, followed by ISO ( $1 \mu \mathrm{M})$ treatment for an additional 48 hours. ${ }^{* *} p<0.01$ vs. ISO+si-NC+Ad-Ctrl and ${ }^{\# \#} p<0.01$ vs. ISO+si-NC+Ad-Jmjd1a. (g) Catalase knockdown blocked JMJD1A-mediated repression of protein synthesis. The cells were treated as in (e). ${ }_{* *} p<0.01 v s$. ISO+si-NC+Ad-Ctrl and ${ }^{\# \#} p<0.01 v s$. ISO+si-NC+Ad-Jmjd1a. (h) Catalase knockdown blocks JMJD1A-mediated repression of the expression of hypertrophic fetal genes. The cells were treated as in ${ }^{* *} p<0.01 v$ s. ISO+si-NC+Ad-Ctrl and ${ }^{\# \#} p<0.01 v s$. ISO+si-NC+Ad-Jmjd1a.

regulate oxidative stress. Our qRT-PCR and western blot evidence demonstrated that JMJD1A expression was downregulated in hypertrophic hearts. To further investigate the roles of JMJD1A, we knockdown the expression or overexpressed it in neonatal rat cardiomyocytes and demonstrated JMJD1A repressed cardiomyocyte hypertrophy induced by ISO. The function of JMJD1A was different from that of JMJD2A because the previous work of Zhang et al. demonstrated that JMJD2A promotes cardiac hypertrophy in response to hypertrophic stimuli in mice [15]. This finding implicates that different members of the JMJD family may have distinctive roles in cardiac hypertrophy.

Elevated total and mitochondrial ROS levels are the fundamental mechanisms underlying the development of cardiomyocyte hypertrophy in vivo and in vitro [5]. Indeed, we observed that JMJD1A was involved in the regulation of total and mitochondrial ROS levels under ISO-induced stress. Importantly, we used the antioxidant regent for total ROS (NAC) or mitochondrial ROS (MitoTEMPO) and observed that either NAC or MitoTEMPO treatment could repress the development of cardiomyocyte hypertrophy. These findings implicate that ROS could be a potential target for the treatment of cardiac hypertrophy. Importantly, we observed that the antioxidants NAC and MitoTEMPO blocked the effects of JMJD1A deficiency on ISO-induced increase in cardiomyocyte size, protein synthesis, and expression of hypertrophic fetal genes.
To further investigate the mechanism underlying the function of JMJD1A in the regulation of total and mitochondrial ROS, we explored the expression of enzymes in ROS production and elimination. We observed that Catalase was most important downstream of JMJD1A. Catalase is a pivotal antioxidant in cytoplasm and mitochondria [30]. Overexpression of Catalase led to the repression of total ROS and mice aging [31]. JMJD1A can promote the expression and enzymatic activity of Catalase in cardiomyocytes. We observed that Catalase was critically involved in the function of JMJD1A because Catalase knockdown blocked the protective function of JMJD1A during cardiac hypertrophy. However, the direct mechanism by which JMJD1A regulates Catalase expression is needed to explore in further study. In addition, other mechanisms may also participate in the function of JMJD1A. For instance, JMJD1A, under hypoxia, promotes the expression of the growth and differentiation factor 15 [32], which functions as a protective and antihypertrophic factor released from the myocardium in association with SMAD protein activation [33].

\section{Conclusion}

In conclusion, we demonstrate that the histone demethylase JMJD1A represses the development of cardiomyocyte hypertrophy by targeting catalase and oxidative stress. 


\section{Data Availability}

The data used to support the findings of this study are available from the corresponding author upon request.

\section{Conflicts of Interest}

The authors declare that they have no conflicts of interest.

\section{Authors' Contributions}

Rongjia Zang and Qingyun Tan contributed equally to this work.

\section{Acknowledgments}

The research was supported by the Health Department of Heilongjiang Province (2019-314).

\section{Supplementary Materials}

"Primers used for quantitative PCR". (Supplementary Materials)

\section{References}

[1] Y. Zhang and J. Ren, "Epigenetics and obesity cardiomyopathy: from pathophysiology to prevention and management," Pharmacology \& Therapeutics, vol. 161, pp. 52-66, 2016.

[2] S. Costantino, P. Libby, R. Kishore, J.-C. Tardif, A. El-Osta, and F. Paneni, "Epigenetics and precision medicine in cardiovascular patients: from basic concepts to the clinical arena," European Heart Journal, vol. 39, no. 47, pp. 4150-4158, 2018.

[3] X.-F. Chen, X. Chen, and X. Tang, "Short-chain fatty acid, acylation and cardiovascular diseases," Clinical Science, vol. 134, no. 6, pp. 657-676, 2020.

[4] S. Samant and M. P. Gupta, Sirtuins as regulators of cardiac hypertrophy and heart failure, Epigenetics in Cardiac Disease, Springer, 2016.

[5] X. Tang, X.-F. Chen, H.-Z. Chen, and D.-P. Liu, "Mitochondrial Sirtuins in cardiometabolic diseases," Clinical Science, vol. 131, no. 16, pp. 2063-2078, 2017.

[6] X. Tang, X. F. Chen, N. Y. Wang et al., "SIRT2 acts as a cardioprotective deacetylase in pathological cardiac hypertrophy," Circulation, vol. 136, no. 21, pp. 2051-2067, 2017.

[7] J. Backs and E. N. Olson, "Control of cardiac growth by histone acetylation/deacetylation," Circulation Research, vol. 98, no. 1, pp. 15-24, 2006.

[8] Y.-X. Luo, X. Tang, X. Z. An et al., "Sirt4 accelerates Ang IIinduced pathological cardiac hypertrophy by inhibiting manganese superoxide dismutase activity," European Heart Journal, vol. 38, pp. ehw138-eh1398, 2016.

[9] W. L. Berry and R. Janknecht, "KDM4/JMJD2 histone demethylases: epigenetic regulators in cancer cells," Cancer Research, vol. 73, no. 10, pp. 2936-2942, 2013.

[10] M. Nakamura and J. Sadoshima, "Mechanisms of physiological and pathological cardiac hypertrophy," Nature Reviews Cardiology, vol. 15, no. 7, pp. 387-407, 2018.

[11] J. J. Saucerman, P. M. Tan, K. S. Buchholz, A. D. McCulloch, and J. H. Omens, "Mechanical regulation of gene expression in cardiac myocytes and fibroblasts," Nature Reviews Cardiology, vol. 16, no. 6, pp. 361-378, 2019.
[12] J. C. Black, C. Van Rechem, and J. R. Whetstine, "Histone lysine methylation dynamics: establishment, regulation, and biological impact," Molecular Cell, vol. 48, no. 4, pp. 491507, 2012.

[13] M. D. Taylor, S. Sadhukhan, P. Kottangada et al., "Nuclear role of WASp in the pathogenesis of dysregulated TH1 immunity in human Wiskott-Aldrich syndrome," Science Translational Medicine, vol. 2, no. 37, p. 37ra44, 2010.

[14] P. C. Chang, L. D. Fitzgerald, D. A. Hsia et al., "Histone demethylase JMJD2A regulates Kaposi's sarcoma-associated herpesvirus replication and is targeted by a viral transcriptional factor," Journal of Virology, vol. 85, no. 7, pp. 32833293, 2011.

[15] Q. J. Zhang, H. Z. Chen, L. Wang, D. P. Liu, J. A. Hill, and Z. P. Liu, "The histone trimethyllysine demethylase JMJD2A promotes cardiac hypertrophy in response to hypertrophic stimuli in mice," The Journal of Clinical Investigation, vol. 121, no. 6, pp. 2447-2456, 2011.

[16] F. A. Mallette, F. Mattiroli, G. Cui et al., "RNF8- and RNF168dependent degradation of KDM4A/JMJD2A triggers 53BP1 recruitment to DNA damage sites," The EMBO Journal, vol. 31, no. 8, pp. 1865-1878, 2012.

[17] L. Fan, F. Zhang, S. Xu et al., "Histone demethylase JMJD1A promotes alternative splicing of AR variant 7 (AR-V7) in prostate cancer cells," Proceedings of the National Academy of Sciences of the United States of America, vol. 115, no. 20, pp. E4584-e4593, 2018.

[18] S. Wilson, L. Fan, N. Sahgal, J. Qi, and F. V. Filipp, “The histone demethylase KDM3A regulates the transcriptional program of the androgen receptor in prostate cancer cells," Oncotarget, vol. 8, no. 18, pp. 30328-30343, 2017.

[19] W. Wan, K. Peng, M. Li et al., "Histone demethylase JMJD1A promotes urinary bladder cancer progression by enhancing glycolysis through coactivation of hypoxia inducible factor 1s," Oncogene, vol. 36, no. 27, pp. 3868-3877, 2017.

[20] Y. Abe, R. Rozqie, Y. Matsumura et al., "JMJD1A is a signalsensing scaffold that regulates acute chromatin dynamics via SWI/SNF association for thermogenesis," Nature Communications, vol. 6, no. 1, 2015.

[21] L. Fan, G. Peng, N. Sahgal et al., "Regulation of c-Myc expression by the histone demethylase JMJD1A is essential for prostate cancer cell growth and survival," Oncogene, vol. 35, no. 19, pp. 2441-2452, 2016.

[22] Q. J. Zhang, T. A. T. Tran, M. Wang et al., "Histone lysine dimethyl-demethylase KDM3A controls pathological cardiac hypertrophy and fibrosis," Nature Communications, vol. 9, no. 1, p. 5230, 2018.

[23] X. Tang, H. Ma, L. Han et al., "SIRT1 deacetylates the cardiac transcription factor $\mathrm{Nkx} 2.5$ and inhibits its transcriptional activity," Scientific Reports, vol. 6, no. 1, 2016.

[24] X.-Z. An, Z. G. Zhao, Y. X. Luo et al., "Netrin-1 suppresses the MEK/ERK pathway and ITGB4 in pancreatic cancer," Oncotarget, vol. 7, no. 17, pp. 24719-24733, 2016.

[25] N. R. Sundaresan, M. Gupta, G. Kim, S. B. Rajamohan, A. Isbatan, and M. P. Gupta, "Sirt3 blocks the cardiac hypertrophic response by augmenting Foxo3a-dependent antioxidant defense mechanisms in mice," The Journal of Clinical Investigation, vol. 119, pp. 2758-2771, 2009.

[26] Y.-H. Loh, W. Zhang, X. Chen, J. George, and H.-H. Ng, "Jmjd1a and Jmjd2c histone H3 Lys 9 demethylases regulate 
self-renewal in embryonic stem cells," Genes \& Development, vol. 21, no. 20, pp. 2545-2557, 2007.

[27] J. Ueda, J. C. Ho, K. L. Lee et al., "The hypoxia-inducible epigenetic regulators Jmjd1a and G9a provide a mechanistic link between angiogenesis and tumor growth," Molecular and Cellular Biology, vol. 34, no. 19, pp. 3702-3720, 2014.

[28] Y. Jiang, S. Wang, Y. Zhao et al., "Histone H3K9 demethylase JMJD1A modulates hepatic stellate cells activation and liver fibrosis by epigenetically regulating peroxisome proliferatoractivated receptor $\gamma$," The FASEB Journal, vol. 29, no. 5, pp. 1830-1841, 2015.

[29] K. Lockman, J. M. Taylor, and C. P. Mack, "The histone demethylase, Jmjd1a, interacts with the myocardin factors to regulate SMC differentiation marker gene expression," Circulation Research, vol. 101, no. 12, pp. e115-e123, 2007.

[30] X. Tang, Y. X. Luo, H. Z. Chen, and D. P. Liu, "Mitochondria, endothelial cell function, and vascular diseases," Frontiers in Physiology, vol. 5, 2014.

[31] D.-F. Dai, L. F. Santana, M. Vermulst et al., "Overexpression of catalase targeted to mitochondria attenuates murine cardiac aging," Circulation, vol. 119, no. 21, pp. 2789-2797, 2009.

[32] A. J. Krieg, E. B. Rankin, D. Chan, O. Razorenova, S. Fernandez, and A. J. Giaccia, "Regulation of the histone demethylase JMJD1A by hypoxia-inducible factor $1 \alpha$ enhances hypoxic gene expression and tumor growth," Molecular and Cellular Biology, vol. 30, no. 1, pp. 344-353, 2010.

[33] J. Xu, T. R. Kimball, J. N. Lorenz et al., "GDF15/MIC-1 functions as a protective and antihypertrophic factor released from the myocardium in association with SMAD protein activation," Circulation Research, vol. 98, no. 3, pp. 342-350, 2006. 